\title{
Modifications regarding diagnostic approach and therapeutic management of urinary stones' disorders during COVID-19 pandemic
}

\author{
Ilias Giannakodimos ${ }^{1} \cdot$ Alexios Giannakodimos ${ }^{1} \cdot$ Evangelos Fragkiadis $^{2} \cdot$ Dimitrios Schizas $^{1} \cdot$ Aikaterini Mastoraki $^{1}$
}

Received: 21 September 2020 / Accepted: 3 November 2020 / Published online: 18 November 2020

c) Springer-Verlag GmbH Germany, part of Springer Nature 2020

\section{Dear Editor,}

A patient presenting with acute colic pain combined with fever and cough constitutes a dilemma for physicians and urologists during COVID-19 period. According to Flammia et al., no alteration in the management of urolithiasis between COVID-19 and no COVID-19 period was reported [1]. These findings may be attributed to the different involvement of each institution in COVID-19 cases, while the treatment pathway in patients with lithiasis during the COVID19 pandemic needs special consideration.

The treatment algorithm must take into account the overall burden of the healthcare system and the prevalence of COVID-19 infections on patients and healthcare workers safety. Modification can include reducing hospital visits, postponing elective cases and treating urgent cases while minimizing chances of exposure to the COVID-19 virus. The need for intervention should be classified according to location and size of the calculi, obstructing symptoms, clinical condition, history and comorbidities of the patient [2]. During COVID-19 period, the surgical removal of obstructed/infected urinary stones should be prevented and only a decompression of the urinary system should be allowed. Decompression management, including JJ stents or nephrostomy, should be performed if possible, outside the operating rooms or at bedside using local anesthesia and sparing ventilators, to avoid COVID-19 transmission [2]. Replacement of functional ureteral stents and nephrostomy tubes with uncomplicated obstruction of upper urinary tract

Aikaterini Mastoraki

dr_kamast@yahoo.gr

1 First Department of Surgery, National and Kapodistrian University of Athens, Laikon General Hospital, 17 Agiou Thoma Street, Athens, Greece

2 First Department of Urology, National and Kapodistrian University of Athens, Laikon General Hospital, Athens, Greece should be postponed for up to 6 months [2]. A telephone evaluation of patients' history combined with meticulous measurement of patients' temperature before hospitalization is necessary to avoid the admission of suspected cases for COVID-19 [3]. Concerning hospitalized patients, a CTscan of the thorax or a nasopharyngeal sample of SARSCoV-2 should always be preceded [3]. Therefore, COVID-19 patients with urinary stones should be subjected to urgent surgical procedures only, in dedicated surgical units followed by strict preventive measures. Finally, telemedicine seems to play an important role in the diagnosis, management and follow-up of patients with uncomplicated urinary stones' disease or history of urolithiasis [3]. A proposed algorithm for the management of patients with urinary stones during the COVID-19 is illustrated in Fig. 1.

The pandemic crisis endures worldwide with different intensity, while temporary treated patients await ultimate treatment. The upcoming challenge of urolithiasis management in COVID-19 era is to schedule the definitive treatment of these patients. Urologic departments should operate cases of urolithiasis, with stent or nephrostomy applied during the pandemic, in relation to the regional epidemiological status, healthcare system resources and safety of patients and healthcare workers. 


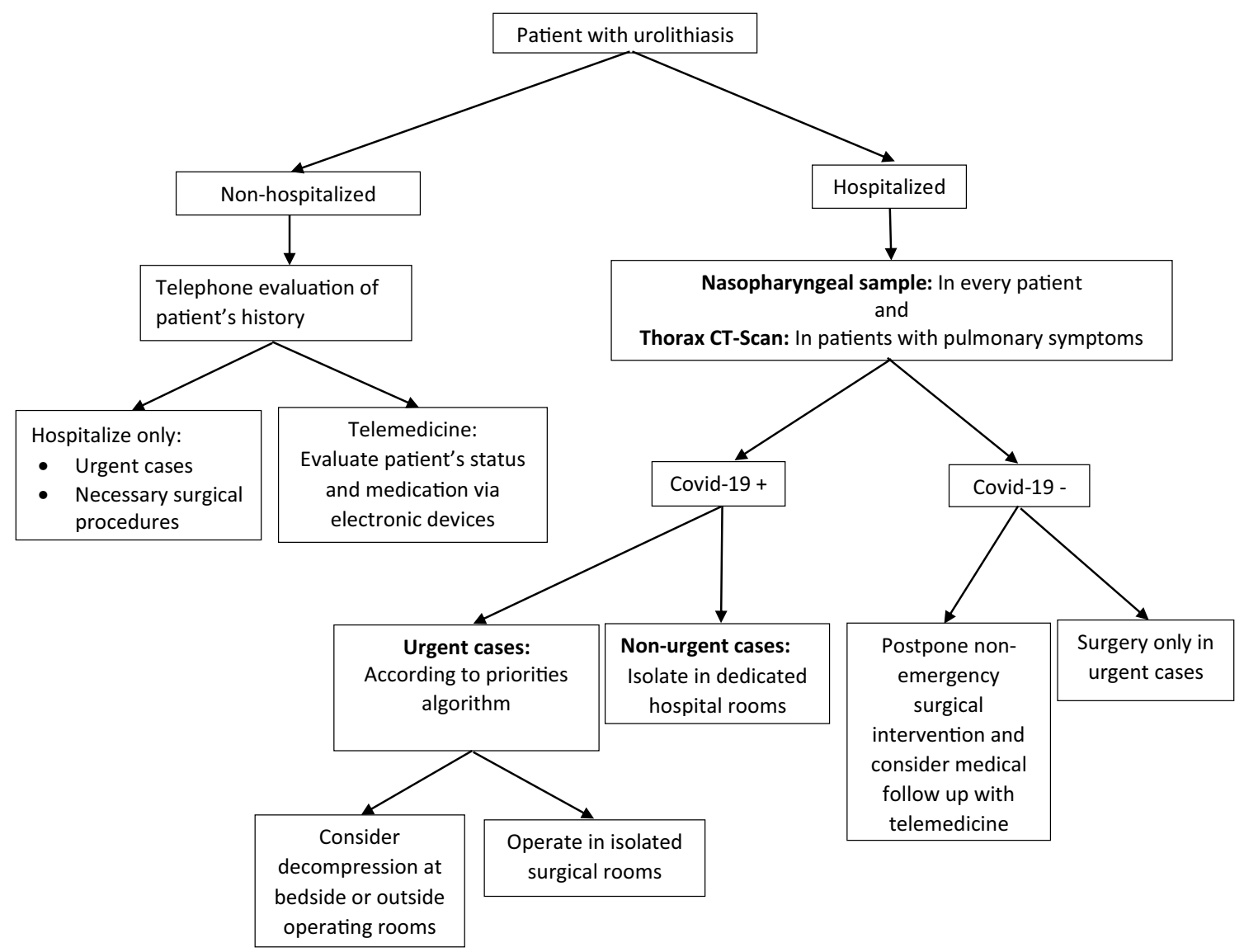

Fig. 1 Algorithm for the management of patients with urinary stones during COVID-19 period

Funding The authors did not receive support from any organization for the submitted work.

\section{Compliance with ethical standards}

Conflicts of interest The authors have no conflicts of interest to declare that are relevant to the content of this article.

\section{References}

1. Flammia S, Salciccia S, Tufano A, Busetto GM, Ricciuti GP, Sciarra A (2020) How urinary stone emergencies changed in the time of COVID-19? Urolithiasis 48:467-469. https://doi.org/10.1007/ s00240-020-01198-3

2. Ribal MJ, Cornford P, Briganti A et al (2020) European association of urology guidelines office rapid reaction group: an organisation-wide collaborative effort to adapt the European association of urology guidelines recommendations to the coronavirus disease 2019 era. Eur Urol 78:21-28. https://doi.org/10.1016/j. eururo.2020.04.056

3. Tefik T, Guven S, Villa L et al (2020) Urolithiasis practice patterns following the COVID-19 pandemic: overview from the EULIS collaborative research working group. Eur Urol 78:e21e24. https://doi.org/10.1016/j.eururo.2020.04.057

Publisher's Note Springer Nature remains neutral with regard to jurisdictional claims in published maps and institutional affiliations. 